\title{
APPROXIMATION OF DISCRETE FUNCTIONS AND SIZE OF SPECTRUM
}

\author{
A. OLEVSKIĬ AND A. ULANOVSKII
}

\begin{abstract}
Let $\Lambda \subset \mathbb{R}$ be a uniformly discrete sequence and $S \subset \mathbb{R}$ a compact set. It is proved that if there exists a bounded sequence of functions in the Paley-Wiener space $P W_{S}$ that approximates $\delta$-functions on $\Lambda$ with $l^{2}$-error $d$, then the measure of $S$ cannot be less than $2 \pi\left(1-d^{2}\right) D^{+}(\Lambda)$. This estimate is sharp for every $d$. A similar estimate holds true when the norms of the approximating functions have a moderate growth; the corresponding sharp growth restriction is found.
\end{abstract}

\section{§1. INTRODUCTION}

1.1. Let $S$ be a compact set in $\mathbb{R}$, and let $m(E)$ denote the Lebesgue measure of $S$. By $P W_{S}$ we denote the Paley-Wiener space

$$
P W_{S}:=\left\{f \in L^{2}(\mathbb{R}): f=\widehat{F}, F=0 \text { on } \mathbb{R} \backslash S\right\}
$$

endowed with the $L^{2}$-norm. Here $\widehat{F}$ stands for the Fourier transform:

$$
\widehat{F}(x):=\int_{\mathbb{R}} e^{i t x} F(t) d t .
$$

Let $B_{S}$ denote the Bernstein space of bounded functions $f$ (with the sup-norm) that are the Fourier transforms of Schwartz distributions supported on $S$. Clearly, every function $f \in P W_{S}$ (and every $f \in B_{S}$ ) can be extended to an entire function of finite exponential type.

Throughout this paper we shall assume that $\Lambda$ is a uniformly discrete set, that is,

$$
\inf _{\lambda, \lambda^{\prime} \in \Lambda, \lambda \neq \lambda^{\prime}}\left|\lambda-\lambda^{\prime}\right|>0 .
$$

The restriction operator

$$
\left.f \rightarrow f\right|_{\Lambda}
$$

is a bounded linear operator from $P W_{S}$ into $l^{2}(\Lambda)$. When this operator is surjective, the set $\Lambda$ is called a set of interpolation for $P W_{S}$. Similarly, if the restriction operator acts surjectively from $B_{S}$ onto $l^{\infty}$, then $\Lambda$ is called a set of interpolation for $B_{S}$. The interpolation problem is to determine when $\Lambda$ is a set of interpolation for $P W_{S}$ or $B_{S}$.

The case of $S=[a, b]$ is classical. Beurling and Kahane proved that in this case the answer can be given essentially in terms of the upper uniform density of $\Lambda$,

$$
D^{+}(\Lambda):=\lim _{r \rightarrow \infty} \max _{a \in \mathbb{R}} \frac{\operatorname{card}(\Lambda \cap(a, a+r))}{r} .
$$

2010 Mathematics Subject Classification. Primary 30D15, 42A16.

Key words and phrases. Paley-Wiener space, Bernstein space, set of interpolation, approximation of discrete functions.

The first author was partially supported by the Israel Science Foundation. 
Namely, in [7, it was shown that the condition

$$
m(S)>2 \pi D^{+}(\Lambda)
$$

is sufficient, while the condition

$$
m(S) \geq 2 \pi D^{+}(\Lambda)
$$

is necessary for $\Lambda$ to be a set of interpolation for $P W_{S}$.

The first condition above is necessary and sufficient for $\Lambda$ to be a set of interpolation for $B_{S}$; see [3].

1.2. The situation becomes subtler for the disconnected spectra. For the sufficiency part, not only the size but also the arithmetical structure of $\Lambda$ is important. On the other hand, Landau 9 proved that (2) is still necessary for $\Lambda$ to be a set of interpolation for $P W_{S}$, for every bounded set $S$.

For a compact spectrum $S$, Landau's result can be stated in a more general form, which requires the interpolation of $\delta$-functions only. For each $\xi \in \Lambda$, let $\delta_{\xi}$ denote the corresponding $\delta$-function on $\Lambda$ :

$$
\delta_{\xi}(\lambda):=\left\{\begin{array}{ll}
1 & \text { if } \lambda=\xi, \\
0 & \text { if } \lambda \neq \xi,
\end{array} \quad \lambda \in \Lambda .\right.
$$

Theorem A [11, Theorem 1]. Let $S$ be a compact set. Suppose there exist functions $f_{\xi} \in P W_{S}$ satisfying $\left.f_{\xi}\right|_{\Lambda}=\delta_{\xi}, \xi \in \Lambda$, and

$$
\sup _{\xi \in \Lambda}\left\|f_{\xi}\right\|<\infty .
$$

Then inequality (2) is fulfilled. The statement is also true for $B_{S}$-spaces.

1.3. The present paper is a direct continuation of [11. We prove that the possibility of approximation of $\delta$-functions on $\Lambda$ with a given $l^{2}$-error already implies an estimate from below on the measure of the spectrum.

Theorem 1. Let $0<d<1$, let $S$ be a compact set, and let $\Lambda$ be a uniformly discrete set. Suppose there exist functions $f_{\xi} \in P W_{S}$ satisfying (3) and such that

$$
\left\|\left.f_{\xi}\right|_{\Lambda}-\delta_{\xi}\right\|_{l^{2}(\Lambda)} \leq d \quad \text { for every } \xi \in \Lambda \text {. }
$$

Then

$$
m(S) \geq 2 \pi\left(1-d^{2}\right) D^{+}(\Lambda) .
$$

Inequality (5) is sharp for every $d$.

Clearly, by letting $d \rightarrow 0$, Theorem 1 implies the necessary condition (2) for interpolation in $P W_{S}$.

In $\S 4$ we prove a version of Theorem 1 for the case where the norms of the approximating functions have a moderate growth. On the other hand, no estimate of the measure of the spectrum is possible if the norms grow too fast.

In contrast to Theorem 1 , in $\S 5$ we show that the possibility of $l^{\infty}$-approximation does not imply any estimate on the measure of $S$. Similar problems for approximation in $l^{p}$ are discussed in $\S 6$.

Some results of this paper were announced in [10.

\section{$\S 2$. LEMMAS}

Our approach to the proof of Theorem 1 includes Landau's method (see [9] and [1, $\S 2])$ and some arguments from Kolmogorov's width theory. 


\subsection{Concentration.}

Definition. Given a number $c, 0<c<1$, we say that a linear subspace $X$ of $L^{2}(\mathbb{R})$ is c-concentrated on a set $Q$ if

$$
\int_{Q}|f(x)|^{2} d x \geq c\|f\|_{L^{2}(\mathbb{R})}^{2} \quad \text { for every } \quad f \in X .
$$

Lemma 1. Given sets $S, Q \subset \mathbb{R}$ of positive measure and a number $0<c<1$, let $X$ be a linear subspace of $P W_{S}$ that is c-concentrated on $Q$. Then

$$
\operatorname{dim} X \leq \frac{m(Q) m(S)}{2 \pi c} .
$$

This lemma is contained in [9] (see statements (iii) and (iv) in Lemma 1).

\subsection{A remark on Kolmogorov's width estimate.}

Lemma 2. Let $0<d<1$, and let $\left\{\mathbf{u}_{j}\right\}, 1 \leq j \leq n$, be an orthonormal basis in an $n$-dimensional complex Euclidean space $U$. Suppose that $\left\{\mathbf{v}_{j}\right\}, 1 \leq j \leq n$, is a family of vectors in $U$ satisfying

$$
\left\|\mathbf{v}_{j}-\mathbf{u}_{j}\right\| \leq d, \quad j=1, \ldots, n .
$$

Then for every $\alpha$, with $1<\alpha<1 / d$, there is a linear subspace $X$ in $\mathbb{C}^{n}$ such that

(i) $\operatorname{dim} X>\left(1-\alpha^{2} d^{2}\right) n-1$;

(ii) the estimate

$$
Q(\mathbf{c}):=\left\|\sum_{j=1}^{n} c_{j} \mathbf{v}_{j}\right\|^{2} \geq\left(1-\frac{1}{\alpha}\right)^{2} \sum_{j=1}^{n}\left|c_{j}\right|^{2}
$$

holds true for every vector $\mathbf{c}=\left(c_{1}, \ldots, c_{n}\right) \in X$.

The classical equality for Kolmogorov's width of "octahedron" (see [8]) implies that the dimension of the linear span of $\mathbf{v}_{j}$ is at least $\left(1-d^{2}\right) n$. This means that there exists a linear space $X \subset \mathbb{C}^{n}$, $\operatorname{dim} X \geq\left(1-d^{2}\right) n$, such that the quadratic form $Q(\mathbf{c})$ is positive on the unit sphere of $X$. Lemma 2 shows that, by a small relative reduction of the dimension, this form can be estimated from below by a positive constant independent of $n$.

We are indebted to E. Gluskin for the following simple proof of this lemma.

Proof. Given an $(n \times n)$-matrix $T=\left(t_{k, l}\right), k, l=1, \ldots, n$, we denote by $s_{1}(T) \geq \cdots \geq$ $s_{n}(T)$ the singular values of this matrix (= the positive square roots of the eigenvalues of $\left.T T^{*}\right)$.

The following properties are well known (see [4, Chapter 3]):

(a) The Hilbert-Schmidt norm of $T$ via singular values:

$$
\sum_{j=1}^{n} s_{j}^{2}(T)=\sum_{k, l=1}^{n}\left|t_{k, l}\right|^{2} .
$$

(b) The minimax principle for singular values:

$$
s_{k}(T)=\max _{L_{k}} \min _{x \in L_{k},\|x\|=1}\|T x\|,
$$

where $\|\cdot\|$ is the norm in $\mathbb{C}^{n}$, and the maximum is taken over all linear subspaces $L_{k} \subseteq \mathbb{C}^{n}$ of dimension $k$.

(c) $s_{k+j-1}\left(T_{1}+T_{2}\right) \leq s_{k}\left(T_{1}\right)+s_{j}\left(T_{2}\right)$ for all $k, j \geq 1, k+j-1 \leq n$. 
Let $T_{1}$ denote the matrix whose columns are the coordinates of $\mathbf{v}_{l}$ in the basis $\mathbf{u}_{k}$, and set $T_{2}:=I-T_{1}$, where $I$ is the identity matrix. Then property (a) and (하) imply that

$$
\sum_{j=1}^{n} s_{j}^{2}\left(T_{2}\right)<d^{2} n,
$$

whence

$$
s_{j}^{2}\left(T_{2}\right) \leq d^{2} \frac{n}{j}, \quad 1 \leq j \leq n .
$$

This and (c) give the inequality

$$
s_{k}\left(T_{1}\right) \geq s_{n}(I)-s_{n-k+1}\left(T_{2}\right) \geq 1-d \sqrt{\frac{n}{n-k+1}} .
$$

Since $s_{n}(I)=1$, setting $k=n-\left[\alpha^{2} d^{2} n\right]$, where [·] means the integral part, we obtain

$$
s_{k}\left(T_{1}\right) \geq 1-\frac{1}{\alpha}, \quad k=n-\left[\alpha^{2} d^{2} n\right] .
$$

Now, property (b) implies that there exists $X$ satisfying the conclusions of the lemma.

\section{§3. Proof of Theorem 1}

3.1. Observe that condition (3) implies the uniform boundedness of the interpolating functions $f_{\xi}$ :

$$
\left|f_{\xi}(x)\right|=\left|\int_{S} F_{\xi}(t) e^{i x t} d t\right| \leq \sqrt{m(S)}\left\|F_{\xi}\right\|_{L^{2}(\mathbb{R})}<C_{1} .
$$

We shall also use the following well-known fact (see [12, Theorem 17]): given a bounded spectrum $S$ and a uniformly discrete set $\Lambda$, there exists $C(S, \Lambda)$ such that

$$
\sum_{\lambda \in \Lambda}|f(\lambda)|^{2} \leq C(S, \Lambda) \int_{\mathbb{R}}|f(x)|^{2} d x \text { for every } f \in P W_{S} .
$$

3.2. Fixing a small number $\delta>0$, we set $S(\delta):=S+[-\delta, \delta]$ and

$$
g_{\xi}(x):=f_{\xi}(x) \varphi(x-\xi), \quad \xi \in \Lambda, \quad \varphi(x):=\left(\frac{\sin (\delta x / 2)}{\delta x / 2}\right)^{2} .
$$

Clearly, $\varphi \in P W_{[-\delta, \delta]}$, so that $g_{\xi} \in P W_{S(\delta)}$. Also, since $\varphi(0)=0$ and $|\varphi(x)| \leq 1, x \in \mathbb{R}$, from (4) it follows that each $\left.g_{\xi}\right|_{\Lambda}$ approximates $\delta_{\xi}$ with an $l^{2}$-error not larger than $d$ :

$$
\left\|\left.g_{\xi}\right|_{\Lambda}-\delta_{\xi}\right\|_{l^{2}(\Lambda)} \leq d, \quad \xi \in \Lambda .
$$

3.3. Fix numbers $a \in \mathbb{R}$ and $r>0$, and set

$$
I:=(a-r, a+r), \quad \nu=\nu(I):=\operatorname{card}(\Lambda \cap I) .
$$

From (1) we have

$$
\nu<C|I|
$$

Here and below in this proof we denote by $C$ constants independent of $I$.

Denote by $\lambda_{1}<\cdots<\lambda_{\nu}$ the elements of $\Lambda \cap I$. Estimate (10) shows that the vectors

$$
\mathbf{v}_{j}:=\left(g_{\lambda_{j}}\left(\lambda_{1}\right), \ldots, g_{\lambda_{j}}\left(\lambda_{\nu}\right)\right) \in \mathbb{C}^{\nu}, \quad j=1, \ldots, \nu,
$$

satisfy (6), where $\left\{\mathbf{u}_{j}, j=1, \ldots, \nu\right\}$ is the standard orthonormal basis in $\mathbb{C}^{\nu}$.

Fix a number $\alpha, 1<\alpha<1 / d$. By Lemma 2 there exists a subspace $X=X(a, r, \alpha) \subset$ $\mathbb{C}^{\nu}$ such that

(i) $\operatorname{dim} X>\left(1-\alpha^{2} d^{2}\right) \nu-1$, and 
(ii) for every vector $\mathbf{c}=\left(c_{1}, c_{2}, \ldots, c_{\nu}\right) \in X$ we have

$$
\left\|\sum_{j=1}^{\nu} c_{j} \mathbf{v}_{j}\right\|^{2}=\sum_{k=1}^{\nu}\left|\sum_{j=1}^{\nu} c_{j} g_{\lambda_{j}}\left(\lambda_{k}\right)\right|^{2} \geq\left(1-\frac{1}{\alpha}\right)^{2} \sum_{j=1}^{\nu}\left|c_{j}\right|^{2} .
$$

Hence, by (8),

$$
\int_{\mathbb{R}}\left|\sum_{j=1}^{\nu} c_{j} g_{\lambda_{j}}(x)\right|^{2} d x \geq C \sum_{j=1}^{\nu}\left|c_{j}\right|^{2}, \quad\left(c_{1}, \ldots, c_{\nu}\right) \in X .
$$

3.4. Set $I^{\prime}:=(a-r(1+\delta), a+r(1+\delta))$. Then, by (7), (9) and (11), every function

$$
g(x):=\sum_{j=1}^{\nu} c_{j} g_{\lambda_{j}}(x)
$$

satisfies the estimate

$$
\begin{aligned}
\int_{\mathbb{R} \backslash I^{\prime}}|g(x)|^{2} d x & =\int_{\mathbb{R} \backslash I^{\prime}}\left|\sum_{j=1}^{\nu} c_{j} f_{\lambda_{j}}(x)\left(\frac{\sin \delta\left(x-\lambda_{j}\right) / 2}{\delta\left(x-\lambda_{j}\right) / 2}\right)^{2}\right|^{2} d x \\
& \leq C\left(\sum_{j=1}^{\nu}\left|c_{j}\right|^{2}\right) \int_{\mathbb{R} \backslash I^{\prime}} \sum_{j=1}^{\nu} \frac{1}{\delta^{4}\left(x-\lambda_{j}\right)^{4}} d x \\
& \leq C|I|\left(\sum_{j=1}^{\nu}\left|c_{j}\right|^{2}\right) \frac{1}{\delta^{4}} \int_{|y|>\delta r} \frac{d y}{y^{4}} \leq \frac{C}{\delta^{7} r^{2}} \sum_{j=1}^{\nu}\left|c_{j}\right|^{2} .
\end{aligned}
$$

Fix $\epsilon>0$. Inequalities (12) and (13) show that there is a number $r_{0}=r(\delta, \epsilon)$ (not depending on $a$ and $\mathbf{c}$ ) such that $r>r_{0}$ implies that

$$
\int_{I^{\prime}}|g(x)|^{2} d x \geq(1-\epsilon) \int_{\mathbb{R}}|g(x)|^{2} d x .
$$

This means that the subspace

$$
G:=\left\{g(x)=\sum_{j=1}^{\nu} c_{j} g_{\lambda_{j}}(x) ;\left(c_{1}, \ldots, c_{\nu}\right) \in X\right\} \subset L^{2}(\mathbb{R})
$$

is $(1-\epsilon)$-concentrated on $I^{\prime}$, provided $r>r_{0}$.

3.5. Since, clearly, $\operatorname{dim} G \geq \operatorname{dim} X$, now Lemma 1 implies that

$$
\operatorname{dim} X \leq \frac{m\left(S_{\delta}\right)\left|I^{\prime}\right|}{2 \pi(1-\epsilon)}
$$

Using inequality (i) for $\operatorname{dim} X$, we obtain

$$
\left(1-\alpha^{2} d^{2}\right) \nu-1 \leq 2 r(1+\delta) \frac{m\left(S_{\delta}\right)}{2 \pi(1-\epsilon)},
$$

whence

$$
\frac{\operatorname{card}(\Lambda \cap(a-r, a+r))}{2 r} \leq \frac{(1+\delta) m\left(S_{\delta}\right)}{2 \pi(1-\epsilon)\left(1-\alpha^{2} d^{2}\right)}+\frac{1}{2 r\left(1-\alpha^{2} d^{2}\right)}
$$

Now, for each fixed number $r$, we choose $a$ so that the left-hand side is maximal and then take the limit as $r \rightarrow \infty$ :

$$
D^{+}(\Lambda) \leq \frac{(1+\delta) m\left(S_{\delta}\right)}{2 \pi(1-\epsilon)\left(1-\alpha^{2} d^{2}\right)} .
$$

Since this inequality is valid for all positive $\epsilon, \delta$ and every $\alpha \in(1,1 / d)$, we conclude that estimate (5) is true. 
3.6. Now we show that estimate (5) is sharp for every $d$. Pick a number $a \in(0, \pi)$, and set $S:=[-a, a], \Lambda:=\mathbb{Z}$, and

$$
f_{j}(x):=\frac{\sin a(x-j)}{\pi(x-j)} \in P W_{S}, \quad j \in \mathbb{Z} .
$$

Then, for every $j \in \mathbb{Z}$, we have

$$
\begin{aligned}
\left\|\left.f_{j}\right|_{\mathbb{Z}}-\delta_{j}\right\|_{l^{2}(\mathbb{Z})}^{2} & =\left\|\left.f_{0}\right|_{\mathbb{Z}}-\delta_{0}\right\|_{l^{2}(\mathbb{Z})}^{2} \\
& =\sum_{k \neq 0}\left(\frac{\sin a k}{\pi k}\right)^{2}+\left(\frac{a}{\pi}-1\right)^{2}=\frac{a}{\pi}-\frac{a^{2}}{\pi^{2}}+\left(\frac{a}{\pi}-1\right)^{2}=1-\frac{a}{\pi} .
\end{aligned}
$$

Hence, the assumptions of Theorem 1 are satisfied with $d^{2}=1-a / \pi$. On the other hand, since $D^{+}(\mathbb{Z})=1$, we see that $m(S)=2 \pi\left(1-d^{2}\right) D^{+}(\mathbb{Z})$, so that estimate (5) is sharp.

\section{§4. Moderate Growth OF NORMS}

4.1. Assume that the norms of functions in (4) satisfy

$$
\left\|f_{\xi}\right\|_{L^{2}(\mathbb{R})} \leq C e^{|\xi|^{\gamma}}, \quad \xi \in \Lambda
$$

where $C$ and $\gamma$ are some positive constants. In this section we show that the statement of Theorem 1 remains true provided $\gamma<1$ and the density $D^{+}(\Lambda)$ is replaced by the upper density $D^{*}(\Lambda)$,

$$
D^{*}(\Lambda):=\limsup _{a \rightarrow \infty} \frac{\operatorname{card}(\Lambda \cap(-a, a))}{2 a} .
$$

The restriction $\gamma<1$ is sharp: we show also that no estimate on the measure of the spectrum is possible if the norms of $f_{\xi}$ grow exponentially.

Observe that $D^{*}(\Lambda) \leq D^{+}(\Lambda)$ for each $\Lambda$. However, $D^{*}(\Lambda)=D^{+}(\Lambda)$ whenever $\Lambda$ is regularly distributed (in particular, when $\Lambda$ is a bounded perturbation of integers).

Theorem 2. Let $0<d<1$.

(i) Suppose $S$ is a compact set and $\Lambda$ a uniformly discrete set. If there exist functions $f_{\xi} \in P W_{S}$ satisfying (4) and (14) with some $0<\gamma<1$, then

$$
m(S) \geq 2 \pi\left(1-d^{2}\right) D^{*}(\Lambda) .
$$

(ii) For every $\epsilon>0$ there is a compact set $S$ with $m(S)<\epsilon$, a sequence $\Lambda=\{n+o(1)\}$, and functions $f_{\xi} \in P W_{S}$ that satisfy (4) and (14) with $\gamma=1$.

Remark 1. Part (i) of Theorem 2 ceases to be true for the density $D^{+}$; see [11, Theorem 2.3].

Remark 2. As in [11, Theorem 2.4], one can check that the assumption $\gamma<1$ in part (i) can be weakened by replacing it with any "nonquasianalytic" growth of norms in (14). It looks likely that the assumption $\gamma=1$ in part (ii) can be replaced with any "quasianalytic" growth. We leave this question open.

Remark 3. Let us show that if $S=[a, b]$ and $D^{-}(\Lambda) \geq 1$, then assumption (4) implies that $b-a \geq 2 \pi\left(1-d^{2}\right)$. Here $D^{-}$is the lower uniform density of $\Lambda$ (to define $D^{-}$, max should be replaced with min in the definition of $D^{+}$).

Recall that a set $\Lambda$ is called a sampling set for $P W_{S}$ if there exist $A, B>0$ such that

$$
A\|f\|_{L^{2}(\mathbb{R})} \leq\left(\sum_{\lambda \in \Lambda}|f(\lambda)|^{2}\right)^{1 / 2} \leq B\|f\|_{L^{2}(\mathbb{R})}
$$


for every $f \in P W_{S}$. The following is a consequence of the classical result of Beurling on sampling sets in Bernstein spaces [2]: Let $\Lambda$ be a uniformly discrete set. If $D^{-}(\Lambda)>a / \pi$, then $\Lambda$ is a sampling set for $P W_{[-a, a]}$, and if $D^{-}(\Lambda)<a / \pi$, then it is not a sampling set for $P W_{[-a, a]}$.

Now, suppose $S=[a, b]$ and $D^{-}(\Lambda) \geq 1$. Then $\Lambda$ is a sampling set for $P W_{[a, b]}$ provided $b-a<2 \pi$. Clearly, in this case, assumption (4) implies (3). By Theorem 1, we conclude that $b-a \geq 2 \pi\left(1-d^{2}\right)$.

Observe that $D^{-}(\Lambda)=1$ for every $\Lambda=\{n+o(1)\}$. It follows that the compact set $S$ in part (ii) of Theorem 2 must be disconnected. On the other hand, we shall see that we can choose $S$ to be a union of two intervals.

4.2. Proof of Theorem 2. The proof of part (i) is quite similar to the proof of Theorem 1.

1. Fix numbers $\delta>0$ and $\beta, \gamma<\beta<1$. There exists a function $\psi \in P W_{(-\delta, \delta)}$ with the following properties:

$$
\psi(0)=1, \quad|\psi(x)| \leq 1, \quad|\psi(x)| \leq C e^{-|x|^{\beta}}, \quad x \in \mathbb{R},
$$

where $C>0$ is some constant. It is well known that such a function can be constructed as a product of $\sin \left(\delta_{j} x\right) /\left(\delta_{j} x\right)$ for a certain sequence $\delta_{j} \rightarrow 0$.

Set

$$
h_{\xi}(x):=f_{\xi}(x) \psi(x-\xi), \quad \xi \in \Lambda .
$$

Then each $h_{\xi}$ belongs to $P W_{S(\delta)}$, and the restriction $\left.h_{\xi}\right|_{\Lambda}$ approximates $\delta_{\xi}$ with an $l^{2}$ error of at most $d$.

2. We set

$$
\Lambda_{r}:=\Lambda \cap(-r, r)
$$

and denote by $C$ various positive constants independent of $r$.

The argument at step 3.3 of the previous proof shows that there exists a linear space $X=X(r)$ of dimension exceeding $\left(1-\alpha^{2} d^{2}\right) \operatorname{card}\left(\Lambda_{r}\right)-1$ and such that

$$
\left\|\sum_{\xi \in \Lambda_{r}} c_{\xi} h_{\xi}(x)\right\|_{L^{2}(\mathbb{R})}^{2} \geq C \sum_{\xi \in \Lambda_{r}}\left|c_{\xi}\right|^{2}
$$

for every vector $\left(c_{\xi}\right) \in X$.

3. Since $\Lambda$ is uniformly discrete, we have $\operatorname{card}\left(\Lambda_{r}\right) \leq C r$. Next, using (14) much as we did with (7), we show that

$$
\left|f_{\xi}(x)\right|^{2} \leq C m(S)\left\|f_{\xi}\right\|_{L^{2}(\mathbb{R})}^{2} \leq C e^{C|\xi|^{\gamma}} \leq C e^{C r^{\gamma}}, \quad \xi \in \Lambda_{r} .
$$

These estimates and (16) imply that

$$
\begin{aligned}
\int_{|x| \geq r+\delta r}\left|\sum_{\xi \in \Lambda_{r}} c_{\xi} h_{\xi}(x)\right|^{2} d x & =\int_{|x| \geq r+\delta r}\left|\sum_{\xi \in \Lambda_{r}} c_{\xi} f_{\xi}(x) \psi(x-\xi)\right|^{2} d x \\
& \leq\left(\sum_{\xi \in \Lambda_{r}}\left|c_{\xi}\right|^{2}\right)\left(C r e^{C r^{\gamma}} \int_{|x|>\delta r} e^{-2|x|^{\beta}} d x\right) .
\end{aligned}
$$

Since $\beta>\gamma$, the last factor tends to zero as $r \rightarrow \infty$. This and the estimate at step 4.2 show that for every $\epsilon>0$ there exists $r_{0}=r(\delta, \epsilon)$ such that the linear space of functions

$$
\left\{h(x)=\sum_{\xi \in \Lambda_{r}} c_{\xi} h_{\xi}(x) ;\left(c_{\xi}\right) \in X\right\}
$$

is $(1-\epsilon)$-concentrated on $(-r-\delta r, r+\delta r)$, for all $r \geq r_{0}$. Moreover, the dimension of this space is at least $\left(1-\alpha^{2} d^{2}\right) \operatorname{card}\left(\Lambda_{r}\right)-1$. 
4. Using Lemma 1, we obtain

$$
m(S(\delta)) \geq \frac{2 \pi(1-\epsilon)}{1+\delta} \frac{\left(1-\alpha^{2} d^{2}\right)(\operatorname{card}(\Lambda \cap(-r, r))-1)}{2 r} .
$$

Now we take the upper limit as $r \rightarrow \infty$ :

$$
m(S(\delta)) \geq \frac{2 \pi(1-\epsilon)}{1+\delta}\left(1-\alpha^{2} d^{2}\right) D^{*}(\Lambda)
$$

Since this inequality is valid for all $\epsilon>0, \delta>0$, and $\alpha \in(1,1 / d)$, we conclude that (15) is true.

5. Here we prove part (ii) of Theorem 2. A union $S$ of two intervals and a small perturbation $\Lambda$ of integers will be chosen as follows:

$$
S:=[-\pi-\epsilon,-\pi+\epsilon] \cup[\pi-\epsilon, \pi+\epsilon], \quad \Lambda:=\left\{n+R^{-|n|-1}, n \in \mathbb{Z}\right\} .
$$

Here $\epsilon>0$ is a given small number and $R>1$.

Denoting by $\lambda_{n}:=n+R^{-|n|-1}$ the elements of $\Lambda$, we set

$$
f_{\lambda_{0}}(x):=\frac{\sin \pi x}{\sin \pi \lambda_{0}} \cdot \frac{\sin \epsilon\left(x-\lambda_{0}\right)}{\epsilon\left(x-\lambda_{0}\right)}
$$

and

$$
f_{\lambda_{n}}(x):=\frac{\sin \pi x}{\sin \pi \lambda_{n}} \cdot \frac{\sin \nu(n)\left(x-\lambda_{n}\right)}{\nu(n)\left(x-\lambda_{n}\right)} \cdot \prod_{|j| \leq 2|n|, j \neq n} \frac{\sin \nu(j)\left(x-\lambda_{j}\right)}{\sin \nu(j)\left(\lambda_{n}-\lambda_{j}\right)}, \quad n \neq 0,
$$

where $\nu(n):=\epsilon /(4|n|+1)$. Since $m(S)=4 \epsilon$, to prove part (ii) it suffices to show that the functions $f_{\lambda_{n}}$ satisfy (4), provided $R$ is sufficiently large.

It is clear that $f_{\lambda_{n}} \in P W_{S}$ and that we have

$$
f_{\lambda_{n}}\left(\lambda_{n}\right)=1, n \in \mathbb{Z}, \quad f_{\lambda_{n}}\left(\lambda_{k}\right)=0, \quad|k| \leq 2 n, \quad k \neq n, \quad n \neq 0 .
$$

Next, we assume that $R$ is so large that the following three estimates hold true for every $n \neq 0$ and every $|k|>2|n|$ :

$$
\left|\frac{\sin \pi \lambda_{k}}{\sin \pi \lambda_{n}}\right| \leq \frac{2 \pi R^{-|k|-1}}{\pi R^{-|n|-1}}=2 R^{-|k|+|n|}, \quad\left|\frac{\sin \nu(n)\left(\lambda_{k}-\lambda_{n}\right)}{\nu(n)\left(\lambda_{k}-\lambda_{n}\right)}\right| \leq \frac{2}{\nu(n)(|k|-|n|)} \leq \frac{8}{\epsilon},
$$

and

$$
\begin{aligned}
\left|\prod_{|j| \leq 2|n|, j \neq n} \frac{\sin \nu(j)\left(\lambda_{k}-\lambda_{j}\right)}{\sin \nu(j)\left(\lambda_{n}-\lambda_{j}\right)}\right| & \leq \prod_{|j| \leq 2|n|, j \neq n} \frac{2}{\nu(j)|j-n|} \\
& \leq\left(\frac{2}{\nu(2 n)}\right)^{4|n|} \frac{1}{|n| !(3|n|) !} \leq\left(\frac{C}{\epsilon}\right)^{4|n|},
\end{aligned}
$$

where $C>1$ is an absolute constant. These estimates imply that

$$
\left|f_{\lambda_{n}}\left(\lambda_{k}\right)\right| \leq 16\left(\frac{C}{\epsilon}\right)^{4|n|+1} R^{-|k|+|n|}, \quad|k|>2|n|, \quad n \neq 0 .
$$

A similar estimate is true for $f_{\lambda_{0}}\left(\lambda_{k}\right)$ for each $k \neq 0$. Clearly, these estimates and (17) prove (4), provided $R$ is sufficiently large. 


\section{$\S 5 . l^{\infty}$-APPROXIMATION}

5.1. In a sharp contrast to Theorem 1 , the possibility of $l^{\infty}$-approximation of $\delta$-functions on $\Lambda$ does not imply any restrictions on the measure of the spectrum.

For the approximation by $P W$-functions this follows from Lemma 3.1 in [1]: For every $N \geq 2$ there exists a set $S(N) \subset(-N, N), m(S(N))=\frac{2}{N}$, such that

$$
\left|\frac{N}{2} \int_{S(N)} e^{i t x} d t-\frac{\sin N x}{N x}\right| \leq \frac{C}{N}, \quad x \in \mathbb{R}
$$

where $C>0$ is an absolute constant.

The function $\sin N x / N x$ is essentially localized in a small neighborhood of the origin, and its Fourier transform is the unit mass uniformly distributed over the interval $[-N, N]$. The above lemma shows that one can redistribute this mass over a set of small measure so that the "uniform error" in the Fourier transform will be $O(1 / N)$.

For the $B_{S}$-functions, the result can be stated in an even stronger form.

Proposition 1. For any number $0<d<1$ and any uniformly discrete set $\Lambda$, there exist a compact set $S$ of measure zero and a bounded sequence of functions $f_{\xi} \in B_{S}$ satisfying

$$
\left\|\left.f_{\xi}\right|_{\Lambda}-\delta_{\xi}\right\|_{l^{\infty}(\Lambda)} \leq d \quad \text { for every } \xi \in \Lambda .
$$

The set $S$ can be chosen depending only on $d$ and the separation constant in (1).

Let us invoke the classical Menshov example from the uniqueness theory for trigonometric series. It can be stated as follows (see [1, Chapter 14, $\S 12, \S 18$, Remark]): There is a singular probability measure $\nu$ with compact support and such that

$$
\widehat{\nu}(x) \rightarrow 0, \quad|x| \rightarrow \infty .
$$

Corollary 1. For every $\epsilon>0$ there is a compact set $S \subset \mathbb{R}$ of Lebesgue measure zero and a function $f \in B_{S}$ such that

$$
f(0)=\|f\|_{L^{\infty}(\mathbb{R})}=1 \quad \text { and } \quad|f(t)|<\epsilon,|t|>\epsilon .
$$

Indeed, it suffices to set $f(x)=\widehat{\nu}(c x)$, where $c$ is sufficiently large.

Now Proposition 1 can be deduced immediately. Indeed, take a positive number $\epsilon<\min \{d, \gamma(\Lambda)\}$, where $\gamma(\Lambda)$ is the infimum in (1). Let $f$ be a function from Colollary 1 . Then the functions $f_{\xi}(x):=f(x-\xi), \xi \in \Lambda$, satisfy the assumptions of Proposition 1.

5.2. Notice that the Bernstein space $B_{S}$ can be defined in a similar way for every unbounded closed spectrum $S$ of finite measure; see [11. In [11] we constructed unbounded spectra $S$ of arbitrarily small measure such that every uniformly discrete set $\Lambda$ is a set of interpolation for $B_{S}$. This was done by a certain iteration argument, by using Lemma 3.1 from that paper. By applying Corollary 1 instead, the same approach can be used for obtaining a more precise version of the result.

Theorem 3. There is a closed set $S$ of measure zero such that every uniformly discrete set $\Lambda$ is a set of interpolation for $B_{S}$.

Remark. The assumption $m(S)=0$ in Proposition 1 and Theorem 3 can be replaced by a stronger metrical "thinness" condition: $S$ may have measure zero with respect to any given Hausdorff scaling function. For such an improvement one needs to use the measures $\nu$ constructed in [5]. 


\section{$\S 6 . B_{S}^{p}$-SPACES AND $l^{p}$-APPROXIMATION}

The spaces $P W_{S}$ and $B_{S}$ can be included into a continuous chain of Banach spaces: given a compact set $S$ and a number $p, 1 \leq p \leq \infty$, denote by $B_{S}^{p}$ the space of all entire functions $f \in L^{p}(\mathbb{R})$ that can be represented as the Fourier transform of a distribution $F$ supported on $S$. Clearly, $B_{S}^{2}=P W_{S}$ and $B_{S}^{\infty}=B_{S}$.

Observe that for $p<p^{\prime}$, we have the embedding

$$
B_{S}^{p} \subset B_{S}^{p^{\prime}}
$$

with the corresponding inequality for norms.

Let $\Lambda$ be a uniformly discrete set. It is well known that the restriction operator $\left.f \mapsto f\right|_{\Lambda}$ acts boundedly from $B_{S}^{p}$ into $l^{p}(\Lambda)$ (see, e.g., [12, p. 82]). $\Lambda$ is called a set of interpolation for $B_{S}^{p}$ if this operator is surjective.

Theorem A implies the following statement.

Theorem 4. Let $S$ be a compact set, and let $p \geq 1$. If there exist functions $f_{\xi} \in B_{S}^{p}$ satisfying $\left.f_{\xi}\right|_{\Lambda}=\delta_{\xi}, \xi \in \Lambda$, and

$$
\sup _{\xi \in \Lambda}\left\|f_{\xi}\right\|_{L^{p}(\mathbb{R})}<\infty
$$

then condition (2) is fulfilled.

In particular, this shows that if $\Lambda$ is a set of interpolation for $B_{S}^{p}$, then estimate (2) is true.

However, when considering $l^{p}(\Lambda)$-approximation by functions in $B_{S}^{p}$, we should distinguish between the following two cases: $1 \leq p \leq 2$ and $2<p \leq \infty$. In the first case, the measure of the spectrum admits an estimate from below as in Theorem 1, while in the second case it does not, as in Proposition 1.

Theorem 5. Let $0<d<1$ and $\Lambda$ be a uniformly discrete set.

(i) Suppose $1 \leq p \leq 2$, and let $S$ be a compact set. If every $\delta_{\xi}, \xi \in \Lambda$, admits the approximation

$$
\left\|\left.f_{\xi}\right|_{\Lambda}-\delta_{\xi}\right\|_{l^{p}(\Lambda)} \leq d, \quad \xi \in \Lambda,
$$

by functions $f_{\xi} \in B_{S}^{p}$ satisfying (19), then condition (5) holds true.

(ii) Suppose $p>2$. There exist a compact set $S \subset \mathbb{R}$ of measure zero and functions $f_{\xi} \in B_{S}^{p}$ satisfying (19) and (20).

Part (i) is a consequence of Theorem 1, the embedding (18), and the standard inequality between $l^{p}$-norms.

Part (ii) follows from a refinement of Menshov's example (see [6]): There is a singular measure $\nu$ with compact support satisfying

$$
\widehat{\nu}(x)=O\left(|x|^{-1 / 2}\right), \quad|x| \rightarrow \infty .
$$

Acknowledgment. A part of the present work was done at the Mathematisches Forschungsinstitut Oberwolfach during a stay within the Research in Pairs Programme, April 2009. The authors appreciate the hospitality of the Institute.

\section{REFERENCES}

[1] N. K. Bari, Trigonometric series, Fizmatgiz, Moscow, 1961; English transl., Bary, N. K., A treatise on trigonometric series. Vols. I, II, The Macmillan Co., New York, 1964. MR.0126115 (23:A3411) MR0171116 (30:1347)

[2] A. Beurling, Balayage of Fourier-Stieltjes transforms, The Collected Works of Arne Beurling. Vol. 2. Harmonic Analysis, Birkhäuser Boston, Inc., Boston, MA, 1989, pp. 341-350. MR1057614 (92k:01046b) 
[3] _ Interpolation for an interval in $\mathbb{R}^{1}$, The Collected Works of Arne Beurling. Vol. 2. Harmonic Analysis, Birkhäuser Boston, Inc., Boston, MA, 1989, pp. 351-365. MR1057614 (92k:01046b)

[4] R. A. Horn and C. R. Johnson, Topics in matrix analysis, Cambridge Univ. Press, Cambridge, 1994. MR1288752 (95c:15001)

[5] O. S. Ivashev-Musatov, M-sets and h-measures, Mat. Zametki 3 (1968), no. 4, 441-447; English transl., Math. Notes 3 (1968), 281-285. MR0227689 (37:3273)

[6] _ On coefficients of trigonometric null-series, Izv. Akad. Nauk SSSR Ser. Mat. 21 (1957), no. 4, 559-578. (Russian) MR0098947 (20:5392)

[7] J.-P. Kahane, Sur les fonctions moyenne-périodiques bornées, Ann. Inst. Fourier (Grenoble) 7 (1957), 293-314. MR0102702 (21:1489)

[8] A. N. Kolmogorov, A. A. Petrov, and Yu. M. Smirnov, A formula of Gauss in the theory of the method of least squares, Izv. Akad. Nauk SSSR Ser. Mat. 11 (1947), no. 6, 561-566. (Russian) MR0023604 (9:381e)

[9] H. J. Landau, Necessary density conditions for sampling and interpolation of certain entire functions, Acta Math. 117 (1967), 37-52. MR0222554 (36:5604)

[10] A. Olevskii and A. Ulanovskii, Interpolation by functions with small spectra, C. R. Math. Acad. Sci. Paris 345 (2007), no. 5, 261-264. MR2353677 (2009b:43009)

[11] _ Interpolation in Bernstein and Paley-Wiener spaces, J. Funct. Anal. 256 (2009), 32573278. MR2504525 (2010c:46066)

[12] R. M. Young, An introduction to nonharmonic Fourier series, Acad. Press, Inc., San Diego, CA, 2001. MR 1836633 (2002b:42001)

School of Mathematics, Tel Aviv University, Ramat Aviv 69978, Israel

E-mail address: olevskii@post.tau.ac.il

Stavanger University, Stavanger 4036, Norway

E-mail address: Alexander.Ulanovskii@uis.no

Received 20/AUG/2009

Originally published in English 